\section{Incidence and biological significance of IKZF1/Ikaros gene deletions in pediatric Philadelphia chromosome negative and Philadelphia chromosome positive B-cell precursor acute lymphoblastic leukemia}

In recent years, there have been a series of contradictory reports based on studies that employed single-nucleotide polymorphism (SNP) arrays regarding the incidence and prognostic significance of IKZF1 deletions in primary leukemic cells from pediatric patients with high-risk B-cell precursor ALL (BPL) ${ }^{1-6}$ Some of the initial reports have proposed that: i) genomic IKZF1 deletions (not alternative splicing) are the cause of expression of dominant-negative IK isoforms; and ii) IKZF1 is a haploinsufficient gene rendering its heterozygous deletions biologically and clinically significant. ${ }^{1,3,4}$ Mullighan et al. reported deletions of IKZF1 in $84 \%$ of Philadelphia chromosome positive $\left(\mathrm{Ph}^{+}\right)$BPL patients, including $76 \%$ of pediatric and $91 \%$ of adult $\mathrm{Ph}^{+}$ $\mathrm{BPL}$ cases. $^{3}$ The same Authors also reported a more than $25 \%$ frequency of $I K Z F 1$ deletions in $\mathrm{Ph}^{-}$high-risk BPL patients. ${ }^{4}$ In both studies, IKZF1 deletions included homozygous/biallelic as well as heterozygous/monoallelic deletion of the entire gene locus as well as intragenic deletions. ${ }^{3,4}$ Likewise, Volejnikova et al. reported discordant results in 206 children with $\mathrm{Ph}^{-}$ALL. ${ }^{7}$ Of 24 patients with overexpression of dominant-negative IK isoforms other than IK6, only one patient had a deletion within the IKZF1 locus and only half of the IK $6^{+}$cases were found to have monoallelic IKZF1 deletions. ' The overall incidence of IKZF1 deletions was only $7 \%$, and no patient had homozygous IKZF1 deletions and no patient had evidence of decreased IK protein expression even in the presence of monoallelic IKZF1 deletions. ${ }^{7}$ Although Mullighan et al. reported IKZF1 deletions as a significant predictor of poor outcome for high-risk BPL patients on the Children's Oncology Group (COG) Study P9906, ${ }^{4}$ a subsequent study by Chen et al. could not confirm the independent prognostic significance of IKZF1 deletions for 499 high-risk BPL patients. ${ }^{5}$ We read with great interest the recent paper of Palmi et al. who reported an elegant study which raises further questions about the biological significance of $I K Z F 1$ deletions in pediatric BPL. ${ }^{6}$ They documented no homozygous IKZF1 deletions and heterozygous IKZF1 deletions were detected in only $\sim 13 \%$ of their $\mathrm{Ph}^{-} \mathrm{BPL}$ patient population. In approximately half of the cases with deletions $(7.1 \%)$, the deletion involved the entire $I K Z F 1$ locus and in the other half a portion of the $I K Z F 1$ gene. ${ }^{6}$ Most importantly, IKZF1 deletions were not an independent prognostic factor of risk of relapse. ${ }^{6}$

In order to gain further insights into the incidence and biological significance of $I K Z F 1$ deletions, we examined the expression levels of $I K Z F 1$ transcripts in primary leukemic cells from 237 pediatric $\mathrm{Ph}^{-} \mathrm{BPL}$ patients in sideby-side comparison with 122 pediatric $\mathrm{Ph}^{+} \mathrm{BPL}$ cases and 74 normal bone marrow specimens in the National Center for Biotechnology Information (NCBI) Gene Expression Omnibus (GEO) database GSE 13159.,

BLAT analysis on IKZF1 target sequences deposited in Affymetrix NetAffx ${ }^{\mathrm{TM}} \quad$ Analysis Center (http://Www.affymetrix.com/analysis/index.affx) mapped the 10 probesets from the Affymetrix Human Genome U133 Plus 2.0 Arrays used in the analysis onto specific IKZF1

A $\quad \operatorname{Normal}(n=74)$

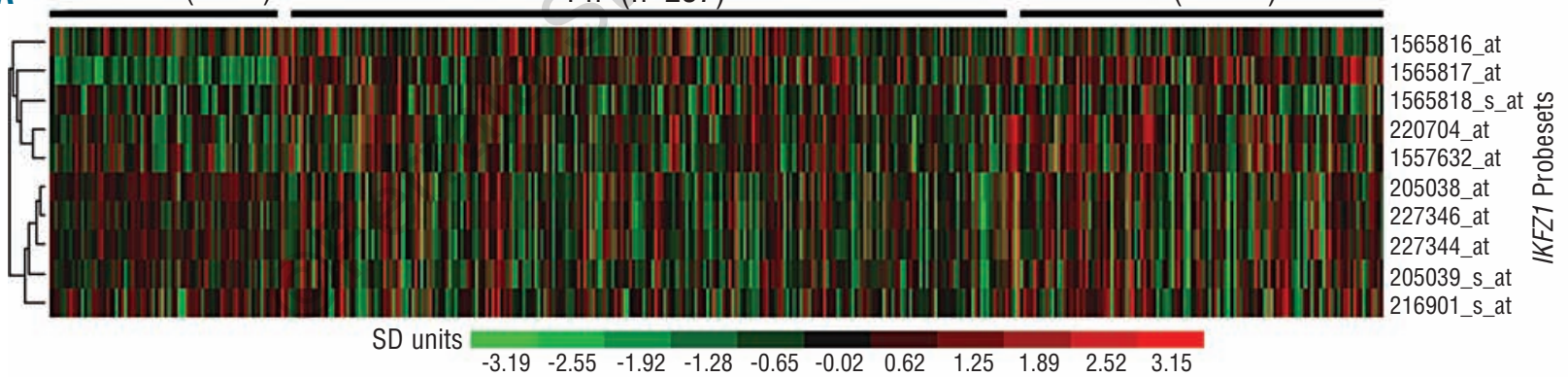

B

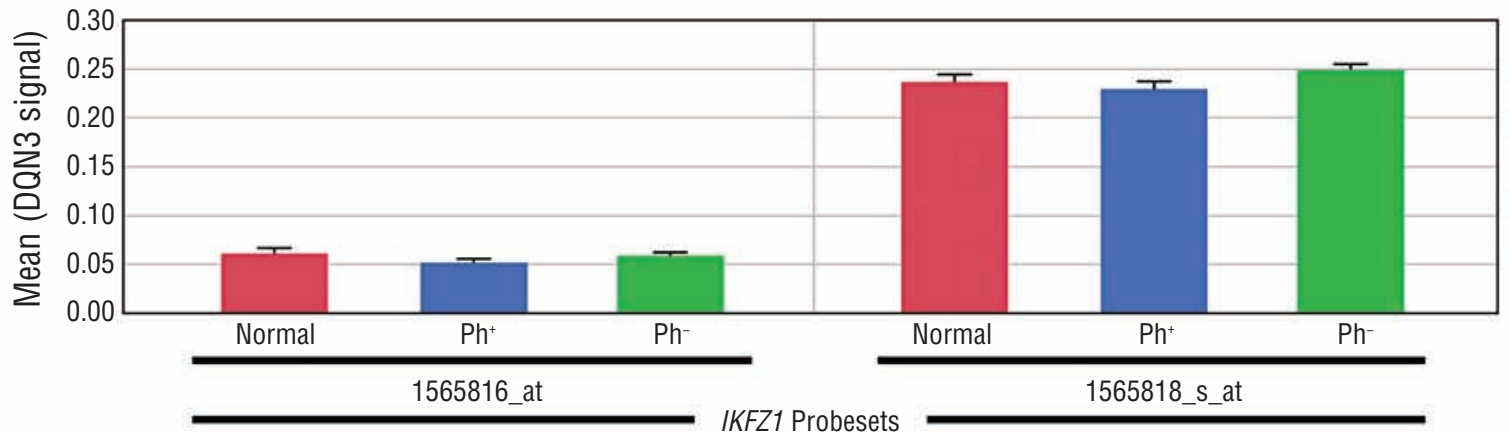

Figure 1. Exon specific expression of IKZF1 in primary leukemic cells from pediatric B-cell precursor ALL (BPL) patients. Exon-specific IKZF1 gene expression levels using 10 IKZF1-specific probesets were compared between primary leukemic cells (GSE13159) from 122 pediatric $\mathrm{Ph}^{+} \mathrm{BPL}$ patients, 237 pediatric, $\mathrm{Ph}^{-} \mathrm{BPL}$ patients and 74 normal bone marrow samples. Heat map depicts up- and down-regulated transcripts ranging from red to green respectively, for standardized DQN3 expression values and clustered according to average distance metric (A). DQN3 normalized expression values for 2 probesets exhibiting specificity for Exon 4 to test for reduction in signal for deletion of exons 4-7 or 2-7 are depicted in the bar chart (B). 
exons visualized using the UCSC genome browser (http://genome.ucsc.edu/cgi-bin/hgBlat? command=start).

The IKZF1 probesets were mapped onto common $I K Z F 1$ deletion regions to identify which probesets would be expected to exhibit reduced gene expression in samples with the reported deletions. Mixed Model Analysis of Variance analysis with three fixed factors ("Diagnosis" (Normal, $\mathrm{Ph}^{-}, \mathrm{Ph}^{+}$, "Probeset"(10 IKZF1 probesets and mean expression of 50 probesets with lowest expression values), an interaction term for Diagnosis $x$ Probeset and a random factor "case" for sample identification was utilized for the analysis of differential IKZF1 gene expression levels. Examination of the Affymetrix probeset coverage in relation to most common IKZF1 microdeletions observed in $\mathrm{Ph}^{+} \mathrm{BPL}$ cases showed that all of these deletions could be detected by multiple IKZF1 probesets. The most common microdeletion occurs between exons 4-7 $(30 \%)^{1}$ that would be detected by 1565816_at and 1565818_s_at followed by exons $2-7(15 \%)^{1}$ that would be detected by 1565816_at, 1565818_s_at, 220704_at, 1557632_at) and large chromosome deletions $(15 \%$; detected 9 of 10 IKZF1 probesets). ${ }^{1}$ The Mixed Model ANOVA explained $90.5 \%$ of the variation in the gene expression data across all 10 $I K Z F 1$ transcripts $24 \%$ variation from the random factor for individual cases and $76 \%$ from the fixed factors. Significant effects of Probeset $\left(F_{10,4300}=3261 ; P<0.0001\right)$, Diagnosis $\left(\mathrm{F}_{2,430}=3.1 ; P=0.046\right)$ and the Interaction term $\left(\mathrm{F}_{20,4300}=13.1 ; P<0.0001\right)$ were observed for these fixed factors). Expression levels for all IKZF1 probesets were greater than the mean expression of the 50 probesets with the lowest expression values (Mean DON3 value $=0.0188$; 95\%CI: 0.0183-0.0192). Our analysis demonstrated no changes in expression that would be expected from homozygous or heterozygous deletions of IKZF1 in primary leukemic cells. In particular, the probesets 1565816_at (specific for exons 1-4) and 1565818_s_at (specific for exon 4 only) did not detect any significantly reduced expression levels in $\mathrm{Ph}^{+}$(Planned Linear Contrasts, $P=0.448$ and $P=0.595$ for 1565816_at and 1565818_s_at respectively) or $\mathrm{Ph}^{-}(P=0.852$ and $P=0.254$ for 1565816_at and 1565818_s_at, respectively, with effect sizes ranging from 0.013 to -0.010 increases in $\mathrm{Ph}^{+} \mathrm{DaN} 3$ normalized expression values) BPL versus normal bone marrow specimens controlling for repeated measures taken from individual cases, that would have suggested heterozygous intragenic deletions between exons 4-7 or exons 2-7.

These results indicate that in pediatric BPL IKZF1 deletions either occur in a minority of leukemic cells in an oligoclonal heterogeneous population of leukemic B-cell precursors or IKZF1 expression is characterized by "allelic imbalance" 10 or "allelic exclusion" ${ }^{11}$ and deletions occur in "inactive" alleles. The reported relationship between IKZF1 deletions detected by SNP arrays and adverse treatment outcome of pediatric BPL is possibly a reflection of underlying genomic instability in aggressive leukemic clones rather than lost or diminished IK function caused by IKZF1 haploinsufficiency, as originally proposed. ${ }^{3,4}$ This would also provide a cogent explanation as to why the prognostic significance of IKZF1 deletions in the Palmi study was markedly enhanced when additional copy number abnormalities involving other genes were present. ${ }^{6}$

\section{Sanjive Qazi, and Fatih M. Uckun}

Children's Center for Cancer and Blood Diseases, Children's Hospital Los Angeles and Department of Pediatrics, University of Southern California Keck School of Medicine, Los Angeles, CA, USA

Correspondence: uckun@usc.edu doi:10.3324/haematol.2013.091140

Key words: IKZF1 deletions, pediatric $P h^{-} B C P-A L L$, copy number abnormalities, prognosis.

Information on authorship, contributions, and financial \& other disclosures was provided by the authors and is available with the online version of this article at www. haematologica.org.

\section{References}

1. Kastner P, Dupuis A, Gaub MP, Herbrecht R, Lutz P, Chan S. Function of Ikaros as a tumor suppressor in B cell acute lymphoblastic leukemia. Am J Blood Res. 2013;3(1):1-13.

2. Dörge P, Meissner B, Zimmermann M, Möricke A, Schrauder A, Bouquin JP, et al. IKZF1 deletion is an independent predictor of outcome in pediatric acute lymphoblastic leukemia treated according to the ALL-BFM 2000 protocol. Haematologica. 2013;98(3):428-32.

3. Mullighan CG, Miller CB, Radtke I, Phillips LA, Ma J, White D, et al. BCR-ABL1 lymphoblastic leukemia is characterized by the deletion of Ikaros. Nature. 2008;453(7191):110-4.

4. Mullighan CG, Su X, Zhang J, Radtke I, Philips LA, Miller CB, et al. Deletion of IKZF1 and Prognosis in Acute Lymphoblastic Leukemia. N Engl J Med. 2009;360(5):470-80.

5. Chen I-M, Harvey RC, Mullighan CG, Gaster-Foster J, Wharton W, Kang $\mathrm{H}$, et al. Outcome modeling with CRLF2, IKZF1, JAK, and minimal residual disease in pediatric acute lymphoblastic leukemia: A Children's Oncology Group Study. Blood. 2012;119(15):3512-22.

6. Palmi C, Valsecchi MG, Longinotti G, Silvestri D, Carrino V, Conter $\mathrm{V}$, et al. What is the relevance of Ikaros gene deletions as prognostic marker in pediatric Philadelphia negative B-cell precursor acute lymphoblastic leukemia? Haematologica. 2013;98(8):1226-31

7. Volejnikova J, Mejstrikova E, Dorge P, Meissner B, Zimmermannova $\mathrm{O}$, Svohgr K, et al. Ikaros (IKZF1) alterations and minimal residual disease at day 15 assessed by flow cytometry predict prognosis of childhood BCR/ABL-negative actute lymphoblastic leukemia. Pediatr. Blood Cancer. 2013;60(3):420-7.

8. Haferlach T, Kohlmann A, Wieczorek L, Basso G, Kronnie GT, Bene $\mathrm{M}-\mathrm{C}$, et al. Clinical utility of micro-array based gene expression profiling in the diagnosis and subclassification of leukemia: report from the International Microarray innovations in leukemia Study Group. J Clin Oncol. 2010;28(15):2529-37.

9. Uckun FM, Ma H, Zhang J, Ozer Z, Dovat S, Mao C, et al. Serine phosphorylation by SYK is critical for nuclear localization and transcription factor function of Ikaros. Proc Natl Acad Sci USA. 2012; 109(44):18072-7.

10. Bryś M, Migdalska-Sęk M, Pastuszak-Lewandoska D, Forma E, Czarnecka K, Domańska D, et al. Diagnostic value of DNA alteration: loss of heterozygosity or allelic imbalance-promising for molecular staging of prostate cancers. Med. Oncol. 2013;30(1):391.

11. Brady BL, Steinel NC, Bassing CH. Antigen receptor allelic exclusion: an update and reappraisal. J Immunol. 2010;185(7):3801-8. 